\title{
Le idee: la struttura fenomenologica e astorica del mondo
}

\author{
As ideias: a estrutura fenomenológica e a-histórica do mundo
}

\author{
Fabio Ciracì \\ Professore dell'Università degli Studi del Salento (Lecce-Italia) \\ E-mail:fabio.ciraci77@gmail.com
}

\begin{abstract}
Riassunto: La Ideenlehre di Schopenhauer si colloca a pieno titolo nel solco della tradizione postkantiana poiché non solo funge da base teoretica alla dottrina del genio romantico e della contemplazione estetica, ma si presta anche ad un'interpretazione in senso illuministico. Difatti, la dottrina delle idee di Schopenhauer è pensata in funzione della volontà nella natura, per la spiegazione scientifica dei fenomeni naturali, come struttura fenomenologica e astorica del mondo, in grado cioè di dare ragione di quei fenomeni, come le leggi e le forze naturali, che non sono individuali o individualizzabili. Allo stesso modo, negli anni Venti del Novecento, Paul Natorp svilupperà la sua metacritica alla dottrina platonica delle idee, con l'intenzione di dare una spiegazione scientifica delle idee non dissimile da quella di Schopenhauer. A conclusione del presente contributo si propone un confronto fra le due posizioni filosofiche.
\end{abstract}

Palavras-chave: Schopenhauer; Ideenlehre; Platone; Natorp.
Resumo: A Ideenlehre de Schopenhauer se apresenta de forma legítima na esteira da tradição pós-kantiana, já que não apenas serve como base teorética para a doutrina do gênio romântico e para a contemplação estética, mas também é passível de uma interpretação de cunho iluminístico. Com efeito, a doutrina das Ideias de Schopenhauer é pensada em função da vontade na natureza, em vista de uma explicação científica dos fenômenos naturais, como estrutura fenomenológica e a-histórica do mundo, em condição de dar conta daqueles fenômenos, tal como as leis e forças naturais que não são individuais ou individualizáveis. Do mesmo modo, nos anos vinte do século XIX, Paul Natorp desenvolveria a sua metacrítica $d a$ doutrina platônica das Ideias com a intenção de oferecer uma explicação científica das Ideias semelhante àquela tentativa de Schopenhauer. Como conclusão da presente investigação, propomos um confronto entre os dois posicionamentos filosóficos.

Keywords: Schopenhauer; Ideenlehre; Platão; Natorp. 


\section{Status quaestionis e prospettive di ricerca}

$\mathrm{I}$

n un precedente contributo $^{1}$ ho provato a esaminare lo sviluppo e la formazione della Ideenlehre schopenhaueriana, analizzando l'occorrenza del filosofema idea all'interno degli scritti dell'autore: dapprima, nei frammenti giovanili e, in seguito, all'interno delle opere pubblicate, e cioè ne Il mondo come volontà e rappresentazione (WWV/MVR) e nei Parerga e paralipomena (P/P), passando brevemente anche per La volontà nella natura $(\mathrm{N} / \mathrm{N})$. L'esame delle varianti semantiche e concettuali ha attestato una pluralità di definizioni che Schopenhauer assegna, di volta in volta, al termine idea, sebbene non sempre tali definizioni siano fra di loro compatibili o sovrapponibili.

Ho cercato inoltre di dimostrare che, all'interno della metafisica della volontà, la dottrina delle idee non deve essere reputata una teoria aporetica, giustapposta al sistema. La Ideenlehre assolve ad un preciso e imprescindibile ruolo ${ }^{2}$, disconosciuto il quale l'intero sistema metafisico schopenhaueriano non funzionerebbe. In terzo luogo, ho proposto di leggere la dottrina delle idee in senso illuministico, ovvero di sottrarla al ruolo esclusivo che la tradizione storiografica le ha da sempre conferito, intestandola unicamente ad una certa interpretazione romantica dell'estetica, considerandola cioè come una sorta di retaggio irrazionalistico dal quale Schopenhauer non sarebbe stato in grado di emanciparsi, una concezione cioè fondata unicamente sul primato del genio e della musica. A partire quindi dai precedenti risultati, cercherò di mostrare che la dottrina estetica delle idee può essere interpretata non solo in senso romantico, come teoria del bello, ma anche in senso illuministico, ovvero come spiegazione scientifica dei fenomeni naturali.

Difatti, una lettura approfondita della Ideenlehre mostra tutta la sua complessità e l'impossibilità di ridurla in toto al canone romantico, cui pure la dottrina delle idee è sicuramente debitrice, per dimostrare che essa non è del tutto ascrivibile all'estetica

${ }^{1}$ Cfr. CIRACÌ, F. Il mondo come volontà, idee e rappresentazione. Per una possibile lettura in senso illuministico della dottrina delle idee (In: Revista Voluntas, Vol. 1, № 1 - 1o sem. 2010), pp. 71-115.

2 Numerose e autorevoli sono invece le voci di chi sostiene la fallacia della dottrina delle idee. Cfr. VECCHIOTTI, I. Introduzione a Schopenhauer, p. 45: «Della volontà, che pure è cosa in sé, lo Schopenhauer conosce un mucchio di cose: fra queste dobbiamo inserire il nucleo, fortemente aporetico costituito dalla teoria delle idee platoniche». Con molta schiettezza, SOSSIO GIAMETTA ha scritto che la dottrina delle idee è da considerarsi «un errore di fondo, dal quale se ne diramano altri» Id., Il mondo di Schopenhauer. Verità ed errori, in A. Schopenhauer, Il mondo come volontà e rappresentazione, pp. V-LXIII, in particolare il I/§2, p. XXXIX.

Le idee: la struttura fenomenologica e astorica del mondo 
romantica di un Wackenroder, di un Jean Paul o di uno Schelling, da cui pure muove e trae ispirazione. Una tale semplificazione sarebbe infatti una banalizzazione della dottrina delle idee. Quella di Schopenhauer è un'interpretazione delle idee platoniche 3 che, collocandosi a pieno titolo nel solco della tradizione postkantiana, si presta ad un'interpretazione anche in senso illuministico, la quale deve di necessità integrarsi con quello romantico, per essere compresa nella sua interezza. La dottrina delle idee è infatti pensata anche in funzione della volontà nella natura, per la spiegazione scientifica dei fenomeni naturali, e quindi in senso scientifico.

Come la Schopenhauer-Forschung internazionale in questi anni ha avuto modo di mostrare, la Ideenlehre schopenhaueriana risente sicuramente di diversi fattori. Si tratta di una dottrina che, nonostante le affermazioni di Schopenhauer, subisce palesemente l'influsso della filosofia di Schelling ${ }^{4}$, in particolare del cosiddetto primo Schelling ${ }^{5}$, e in relazione alla nostra indagine è debitrice della schellinghiana dottrina delle potenze. Come ha scritto Ernst Cassirer:

Non è questa la prima volta che la dottrina platonica delle idee interviene, nella sua particolare applicazione al problema dell'arte, come

\footnotetext{
${ }^{3}$ Sulla storia delle interpretazioni della dottrina delle idee di Platone nell'Ottocento, si veda l'ottimo lavoro di ASMUTH, C. Interpretation - Transformation, Das Platonbild bei Fichte, Schelling, Hegel, Schleiermacher und Schopenhauer und das Legitimationsproblem der Philosophiegeschichte, in particolare il cap. 6. Platon als Vorläufer Kants: Schopenhauer, p. 245 e ss. Sul platonismo di Schopenhauer si era già espresso, in particolare, anche ZINT, H. Schopenhauer und Platon, pp. 17-41, soprattutto pp. 20-29.

${ }^{4}$ Della sterminata bibliografia su questo tema, si vedano soprattutto: SEGALA, M. Schopenhauer, la filosofia, le scienze, p. 49 e ss. Sull'origine della concezione delle idee in Schopenhauer e sul rapporto con Platone, cfr. HÜBSCHER, A. Arthur Schopenhauer: un filosofo controcorrente, pp. 111-112 e KAMATA, Y. Der junge Schopenhauer, pp. 115-118. Su Schelling-Schopenhauer: Schopenhauer-Handbuch. Leben-WerkeWirkung, hrsg. v. SCHUBBE, D.; KOßLER, M., in relazione alle fonti; cfr. INVERNIZZI, G. Invito al pensiero di Schopenhauer, passim; KOßLER, M. Substantielles Wissen und subjektives Handeln dargestellt in einem Vergleich von Hegel und Schopenhauer, passim; Die Ethik Arthur Schopenhauers im Ausgang vom deutschen Idealismus (Fichte/Schelling), hrsg. v. HÜHN, L. Anche i primi interpreti di Schopenhauer, soprattutto Eduard von Hartmann, vedevano più affinità che differenze nelle dottrine dei due filosofi, e ne tentarono una sintesi. Di grande rilievo l'interpretazione della dottrina delle idee proposta da Hartmann, come enti mediatrici; cfr. HARTMANN, E. Philosophie des Unbewussten.

${ }^{5}$ Nella biblioteca di Schopenhauer, conservata (e in gran parte ricostruita) presso lo Schopenhauer-Archiv di Francoforte, è possibile consultare i seguenti volumi di Schelling: dagli articoli usciti per gli Jahrbücher der Medecin als Wissenschaft e per la Zeitschrift für spekulative Physik, alle Ideen zu einer Philosophie der Natur, la Weltseele, Bruno, System des transzendentalen Idealismus e il primo volume delle Philosophische Schriften (Landschut, Krüll, 1809). Il primo volume consta dei seguenti saggi: Vom Ich als Prinzip der Philosophie, oder über das Unbedingte im menschlichen Wissen (1795), Philosophische Briefe über Dogmatismus und Kritizismus (1795), Abhandlungen zur Erläuterung des Idealismus der Wissenschaftslehre (1796), Ueber das Verhältniss des bildenden Künste zu der Natur (1807), e Philosophische Untersuchungen über das Wesen der menschlichen Freiheit (1809). I volumi di Schelling posseduti da Schopenhauer presentano glosse, sottolineature e numerose critiche di pugno di Schopenhauer. Come scrive Segala, «La lettura di quegli scritti non avvenne secondo il loro ordine cronologico. Nel 1810, Schopenhauer prese a prestito dalla biblioteca di Gottinga Ideen (1797) e Weltseele (1798)». L'elenco degli scritti schellinghiani posseduti da Schopenhauer è reperibile in HN, V, pp. 143-149, 189, 263-264.

Le idee: la struttura fenomenologica e astorica del mondo
} 
motivo determinante nello sviluppo della speculazione postkantiana. È stato rilevato giustamente che, rispetto all'insieme di questa speculazione, in particolare alle dottrine di Fichte e Schelling, Schopenhauer non si pone affatto con quell'indipendenza di cui si potrebbe aver l'impressione dai continui ed aspri stacchi di cui le fa segno. A questo proposito è ben difficile, in particolare, che sussistano dubbi sul fatto che egli si ricolleghi a quella concezione della dottrina che Schelling aveva esposta nel dialogo Bruno e nel discorso Sul rapporto delle arti figurative con la natura. Come Schelling, anch'egli si rifà, più che a Platone, a Plotino e alla dottrina plotiniana del bello intelligibile 6 .

Altri studiosi hanno riconosciuto nella dottrina delle idee di Schopenhauer la presenza della filosofia di Agostino, oppure - come già Cassirer - di Plotino e dei mistici medievali. Alcuni ancora hanno rilevato come la Ideenlehre sia condizionata dalla lezione di Goethe, attraverso il tema faustiano delle grandi madri e la lettura goethiana della filosofia di Spinoza ${ }^{8}$. E a me pare che questo tema non sia stato ancora del tutto scandagliato a fondo e che meriterebbe, in futuro, un maggior approfondimento. Infine, recenti studi sulla formazione di Schopenhauer ${ }^{9}$ mostrano il peso che la traduzione tedesca dei Werke di Platone ad opera di Schleiermacher ${ }^{10}$ e la Geschichte der Philosophie di Tennemann hanno avuto sul giovane filosofo, condizionandone non solo l'interpretazione della filosofia kantiana ${ }^{11}$, e più in generale la genesi e l'ideazione complessiva del sistema filosofico, ma anche il lessico filosofico.

Sin qui, a grandi linee, le principali linee di ricerca. Con il presente saggio non si

${ }^{6}$ CASSIRER, E. Storia della filosofia moderna, pp. 264-265.

7 Si vedano in particolare: MOLLOWITZ, G. Die Assimilation der platonisch-augustinischen Ideenlehre durch Schopenhauer, pp. 131-151. KAMATA, Y. Platonische Idee und die anschauliche Welt bei Schopenhauer, pp. 84-93.

$8 \mathrm{Su}$ questo tema ha insistito BARBERA, S. Il mondo come volontà e rappresentazione di Schopenhauer: introduzione alla lettura, pp. 125-126. Barbera sostiene che la concezione schopenhaueriana dell'idea va ricondotta a Spinoza e Goethe, più che a Platone.

${ }_{9}^{9} \mathrm{Mi}$ riferisco in particolare alle innovative ricerche, ancora in corso, di NOVEMBRE, A. Cfr. Id., Il giovane Schopenhauer e Fichte: la duplicità della coscienza, Dissertatio, consultabile all'indirizzo <http://ubm.opus.hbz-nrw.de/volltexte/2014/3685/pdf/doc.pdf>.

10 PLATO, Werke, aus dem Griechischen übersetzt von SCHLEIERMACHER, F., V, 1804-1810; VI, 18171828. Platonis philosophi quae exstant Graece ed editionem Henrici Stephani accurate expressa cum Marsilii Ficini interpretatione. Praemittitur L. III Laertii de vita et dogmatibus Platonis cum notitia literaria. Accedit varietas lectionis, Stud. Soc. Bip. Biponti, ex Typographia Societatis, I - XII, 1781-1787. Le indicazioni dei prestiti sono ricavate da Arthur Schopenhauers sämtliche Werke, p. 105.

11 NOVEMBRE, A. Platonische Idee und Ding an sich. Der junge Schopenhauer als Leser von Tennemanns Geschichte der Philosophie (in print). Cfr. D’ALFONZO, M. (hrsg. v.), Schopenhauers Kollegnachschriften der Metaphysik und Psychologievorlesungen von G. E. Schulze, cit., p. 101, nota. Uno studio più approfondito dell'influenza che la filosofia elementare di Karl Leonhard Reinhold e poi di "Enesidemo" Schulze hanno avuto sul giovane Schopenhauer si trova in Yasuo Kamata ma è ancora certamente un tema da approfondire e rivalutare.

Le idee: la struttura fenomenologica e astorica del mondo 
intende però discutere della dottrina delle idee di Schopenhauer alla luce delle fonti cui essa è riconducibile, il che è certamente presupposto come requisito essenziale, a meno di non stabilire arbitrari paragoni fra la filosofia di Schopenhauer e il pensiero di altri filosofi. L'importanza della ricostruzione storica e dell'analisi delle fonti schopenhaueriane non è posta qui in discussione: essa non solo è incontestabile ma necessaria. Tuttavia, si può correre il rischio di ridurre la filosofia schopenhaueriana al suo contesto, di vivisezionarla in parti isolate, conoscendo meticolosamente i singoli organi, ma smarrendone il punto di vista generale, la sua organicità, il suo contributo specifico e, soprattutto, la sua portata innovativa.

In questa sede preme allora interrogarsi sulla novità della proposta ermeneutica schopenhaueriana della dottrina delle idee e comprendere se e in base a quali presupposti è possibile collocarla nel solco della tradizione postkantiana ${ }^{12}$.

L'intento è quindi di dimostrare la complessità della Ideenlehre schopenhaueriana intesa, da un lato, come struttura fenomenologica e, dall'altro, astorica del mondo, in grado di dare ragione di quei fenomeni naturali che non sono individuali o individualizzabili, poiché essi non cadono sotto il principium individuationis e quindi non possono essere spiegati attraverso la quadruplice radice del principio di ragion sufficiente ${ }^{13}$.

\footnotetext{
12 Sull'interpretazione della dottrina delle idee di Platone da parte di Schleiermacher in relazione al dibattito postkantiano, cfr. ARNDT, A. Friedrich Schleiermacher als Philosoph, Teil V: Antike Philosophie, § 1 Schleiermacher und Platon: p. 269: «Platon dagegen habe die oberste Wissenschaft als den gemeinschaftlichen Grund der Ethik und Physik nur poetisch bezeichnet. Gleichwohl erhellt aus dieser Bezugnahme, in welcher Weise Schleiermacher systematisch an Platon anknüpfen will: die endliche, im Werden begriffene und unter dem Gegensatz stehende „Welt" soll unmittelbar auf das Unwandelbare und Unendliche ihres Einheitsgrundes so bezogen werden, dass die Besonderungen der erscheinenden Wirklichkeit als individualisierte Darstellungen dieser "Idee“ gelten. Platon steht hier als Zeuge für die Annahme eines unmittelbaren, absolutidentischen Seins, welches den Entgegensetzungen der endlichen Wirklichkeit voraus- und zugrunde liegt. In dieser Konzeption, die sich den Debatten der nachkantischen Philosophie in Deutschland verdankt, sind Kantisch-Fichtesches Transzendentalsubjekt und Spinozistische Substanz zu einem bewusstseinstranszendenten Einheitsgrund zusammengeschmolzen. Der Sache nach wird nur die Position bekräftigt, die Schleiermacher bereits 1793/94 in der Konfrontation Kants mit Spinoza erreicht hatte. Ihre Verknüpfung mit der Platonischen Ideenlehre führt zu keiner tiefgreifenden Modifikation und bleibt insofern äußerlich. Als Bezugspunkt fungiert dabei ein wenig spezifiziertes, ästhetisch gerichtetes Verständnis des Platonismus, das über die bezeugte Verehrung für den Geist Platons hinaus kein systematisches und kritisches Eingehen auf die Sache und die theoretischen Mittel seiner Philosophie selbst erkennen lässt». Sul tema si vedano anche: GADAMER, H.-G., „Schleiermacher als Platoniker“, 141-149; KRAPF, ADOLF, G. Platonic Dialectics and Schleiermacher's Thought: an Essay towards the Reinterpretation of Schleiermacher; POHL, K. Studien zur Dialektik Friedrich Schleiermachers; SCHOLTZ, G. Schleiermacher und die Platonische Ideenlehre, 849-871; MORETTO, G. „Platonismo e romanticismo. Platone nei Discorsi sulla religione di Schleiermacher“, pp. 233-269.

${ }^{13}$ MALTER, R. Der eine Gedanke. Hinführung zur Philosophie Arthur Schopenhauer, p. 62: "Qualitativ sind sie [die Ideen] daher radikal von den Erscheinungen getrennt, in denen sie sich in zahlenmäßiger Mannigfaltigkeit zum Ausdruck bringen: „Die Idee hingegen geht in jenes Princip nicht ein: daher ihr Le idee: la struttura fenomenologica e astorica del mondo
} 
A questo scopo, sarà innanzitutto necessario cominciare velocemente con l'evidenziare le analogie e le differenze sostanziali che intercorrono fra la dottrina delle idee di Platone e quella di Schopenhauer, per approfondire maggiormente le caratteristiche delle Ideenlehre schopenhaueriana e riconoscerne la sua peculiarità.

Si proverà infine a confrontare la Ideenlehre schopenhaueriana con la teoria delle idee sostenuta nella prima metà del Novecento da Paul Natorp, mostrandone i numerosi punti di affinità e l'esistenza di una linea di continuità fra le due proposte teoriche, le quali si iscrivono entrambi nel solco della tradizione kantiana e ne tracciano gli assi storici fondamentali.

\section{Platone, Schopenhauer e il mondo delle idee}

Come è noto, per Platone, le idee sono essenze immutabili (Repubblica, VI, 484cd) dell'iperuranio (Fedone, 75 C 10-D 3:), entità etiche (idee-valore) e matematiche, modelli (Parmenide, 132d) ${ }^{14}$, essenze ordinate gerarchicamente e culminanti nell'idea di sommo bene. Appartenendo al mondo dell'essere, per Platone, le idee non divengono e non sono soggette ad alcuna azione, sono invece gli oggetti sensibili del divenire che si relazionano alle idee in tre modi: mimesi, metessi e parusia. In quanto essenze ultime della realtà, le idee sono arché, principii, sono in sé e per sé (nel Fedone, $\alpha u ̛ \tau o ̀ ~ \kappa \alpha \theta^{\prime} \alpha u ̛ \tau o ́$ ) e non sono quindi derivabili ma sono esse stesse le matrici del mondo. Come è evidente anche a un primo sguardo, le differenze che intercorrono fra le idee di Platone e quelle descritte da Schopenhauer sono numerose e fondamentali. Vi è in Platone una differenza fra essere delle idee e mondo della sensibilità che è ad un tempo alternativa ed esclusiva, e non ha un termine medio. Per Schopenhauer, invece, le idee si trovano sì nel fenomeno,

weder Vielheit noch Wechsel zukommt. Wahrend die Individuen, in denen sie sich darstellt, unzählige sind und unaufhaltsam werden und vergehn, bleibt sie unverändert als die eine und selbe stehn, und der Satz vom Grunde hat für sie keine Bedeutung" (W I, S. 200)».

14 PLATO. Parmenide, 132d: «Mi sembra assai preferibile assumere che le Idee stanno alla realtà come modelli e gli altri oggetti assomigliano ad esse e sono copie. La partecipazione alle Idee delle altre cose non consiste in altro che nell'essere fatte a immagine di quelle [delle idee]». Com'è noto, la cosiddetta "versione classica" della teoria delle idee (cfr. VEGETTI, M. Quindici lezioni su Platone, Einaudi, p. 156) è esposta da Platone nel Fedone 95b-108c e ne La Repubblica VI, 507a-b, VII e X, 596a, per essere ampliata, con alcune variazioni, significativamente nel Parmenide 128-136e e nel Sofista, 246b, 248a. Sul tema, si veda anche la celebre 7 lettera $342 \mathrm{a}-344 \mathrm{~d}$. È utile citare anche le definizioni sintetiche, e la relativa spiegazione, con cui Reale caratterizza l' idea platonica: intelligibilità, incorporeità, l'essere in senso pieno, l'immutabilità, la perseità, l'unità, cfr. REALE, G. Per una nuova interpretazione di Platone alla luce delle "dottrine non scritte", pp. 166ss.

Le idee: la struttura fenomenologica e astorica del mondo 
ma sono medie fra la volontà in sé e i singoli oggetti. Difatti, per Schopenhauer, le idee non sono l'essenza della realtà, che è invece la Volontà metafisica universale, ma esse sono invece la forma generale del fenomeno. Le idee schopenhaueriane sono immanenti alla realtà, e non come per Platone trascendenti, ed esistono in quanto si pongono di fronte alla volontà metafisica in sé, non ancora obiettivata, come di fronte ad uno specchio, rappresentandone l'opposto: le idee sono fenomeno generale e pertanto toto coelo diverse dal Wille an Sich. Non vi è dunque alcun rapporto di imitazione, partecipazione o presenza della volontà in sé, come aveva inteso Platone - a meno di non interpretare la mimesi come una relazione analogica (il che è invece presupposto implicitamente, ma sviluppato in seguito dalla corrente plotiniana).

Il processo di determinazione delle idee in Schopenhauer sembra seguire in parte, ancora una volta, la lezione fichtiana della creazione del non-Io della Dottrina della scienza: la Volontà metafisica è in tutto ed è il tutto, è attività pura, come l'Io Assoluto fichtiano il quale, per poter agire e conoscersi (principio etico e gnoseologico), ha bisogno di un oggetto della sua azione, ovvero un non-Io, sicché è costretto ad autolimitarsi. Tale oggetto è posto di fronte a sé, non già nel tempo ma simultaneamente alla Volontà in sé. E se la volontà in sé15 è l'essenza pura della realtà che ancora non si è manifestata nel mondo, allora le idee sono il fenomeno nella sua purezza, il perfetto fenomeno, «oggettità immediata e adeguata [unmittelbare und adäquate Objektität]»16.

Dal canto suo, Schopenhauer sa bene di muoversi su un crinale ermeneutico sottile e, nel reinterpretare Platone, di allontanarsene significativamente:

L'idea platonica è necessariamente oggetto, un che di cognito, una

15 Schopenhauer distingue chiaramente «l'in sé della volontà», come egli lo chiama [SCHOPENHAUER, A. WWV I/MVR I, § 34, pp. 366-367], dalla sua manifestazione oggettiva, l'idea. È fondamentale sottolineare che per Schopenhauer la Volontà metafisica intesa come Volontà in sé non coincide pienamente con il mondo, con la sua oggettivazione, con il Weltwille, con la volontà che si determina come mondo, ma in realtà lo esubera: «[Che] per me il mondo non esaurisce ogni possibilità dell'essere e [che] in questa rimane ancora molto spazio per ciò che noi indichiamo solo negativamente come la negazione della volontà di vivere», in SCHOPENHAUER, A. WWV II/MVR II, capitolo 50, «Epifilosofia», pp. 2198-9.

16 SCHOPENHAUER, A. WWV I/MVR I, § 31, pp. 348-349: «Spero inoltre che [...] non si esiterà a riconoscere, nei determinati gradi di oggettivazione di questa volontà costituente l'in sé del mondo, quelle che Platone chiamava le idee eterne, ovvero le forme immutabili (idee), [...]./ Se dunque la volontà è per noi la cosa in sé e l'idea invece l'oggettità immediata di quella volontà in un grado determinato, troviamo

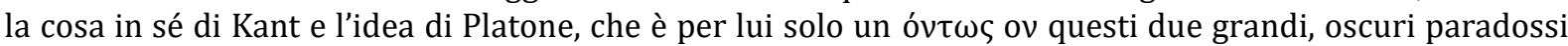
dei due massimi filosofi dell'Occidente - certo non come identiche, ma ad ogni modo come strettamente apparentate e distinte da un'unica determinazione». Id., § 32, pp. 356-7. In realtà, Schopenhauer puntualizza anche cosa differenzia la sua idea da quella del divino Platone: «L'idea platonica è necessariamente oggetto, un che di cognito, una rappresentazione, e appunto per ciò, ma anche solo per ciò, diversa dalla cosa in sé».

Le idee: la struttura fenomenologica e astorica del mondo 
rappresentazione, e appunto per ciò, ma anche solo per ciò, diversa dalla cosa in sé17,

la quale invece è inconoscibile ovvero impossibile da concepire con la nostra ragione. Tant'è che il filosofo scrive,

l'idea e la cosa in sé, nonostante ogni intima concordanza fra Kant e Platone e l'identità del fine che entrambi avevano in mente, [...] non sono per noi semplicemente una sola e medesima cosa. Al contrario, l'idea è per noi solo l'oggettità immediata e quindi adeguata della cosa in sé, che essa stessa è però la volontà, la volontà in quanto non è ancora oggettivata, non è ancora divenuta rappresentazione ${ }^{18}$.

Né va trascurato che per Schopenhauer non vi è coincidenza fra idee e forme apriori individuali nel senso assegnate a quest'ultime da Kant, poiché Kant riferisce sempre le forme a priori quam experientia ad un individuo conoscente:

Si andò dietro alle parole, alle parole: «rappresentazioni a priori, forme dell'intuire e del pensare conosciute indipendentemente dall'esperienza, concetti originari dell'intelletto puro» ecc. - e ci si chiese poi se le idee di Platone, che sarebbero appunto anche concetti originari e poi ancora reminiscenze di una contemplazione precedente alla vita delle cose dotate di essenza verace, non fossero per caso la stessa cosa delle forme dell'intuire e del pensare di Kant, che si trovano a priori nella nostra coscienza. Queste due dottrine del tutto eterogenee: quella kantiana delle forme, che limitano la conoscenza dell'individuo al fenomeno, e quella platonica delle idee, la conoscenza delle quali appunto nega espressamente quelle forme, - queste dottrine, in tal senso diametralmente opposte, per il fatto che si somigliano un po' nei loro termini, le si paragonò attenta- mente, si discusse e si disputò sulla loro identità, finché poi si trovò che in realtà non erano la stessa cosa, e si concluse che la dottrina delle idee di Platone e la critica della ragione di Kant non avevano nessuna concordanza ${ }^{19}$.

Ed è proprio per evitare ogni fraintendimento con la teoria gnoseologica kantiana

\footnotetext{
17 SCHOPENHAUER, A. WWV I/MVR I, § 32, pp. 356-7.

18 SCHOPENHAUER, A. WWV I/MVR I, § 32, pp. 356-7. Per Schopenhauer, che qui segue e radicalizza la lezione di Kant, vi è uno scarto incolmabile fra essere e conoscenza. Tutt'altra la posizione platonica. Come ha segnalato Reale: «Il cespite teoretico di questa dottrina [dottrina delle idee matematiche] è da ricercarsi nella convinzione radicalissima in Platone della corrispondenza strutturalmente perfetta fra conoscere ed essere («la stessa cosa è il conoscere e l'essere» Parmenide, fr. 3, Diels-Kranz), per cui ad un determinato livello di conoscenza di un determinato tipo deve necessariamente far riscontro un corrispettivo livello di essere», in REALE, G. Per una nuova interpretazione di Platone, cit., p. 238.

19 SCHOPENHAUER, A. WWV I/ MVR I, § 31. Come esplicita in nota Schopenhauer, il riferimento a tali interpretazioni è Immanuel Kant, un monumento di Fr. Bouterweck, p. 49, - e la Storia della filosofia di Buhle, voi. $6^{\circ}$, pp. $802-815$ e 823.

Le idee: la struttura fenomenologica e astorica del mondo
} 
degli a priori che, probabilmente, Schopenhauer non utilizza mai l'espressione "a priori naturale" per definire le idee, poiché l'apriori richiama sempre la dimensione individuale.

Il processo di oggettivazione dei fenomeni, inoltre, implica una dimensione temporale, alla quale le idee si sottraggono per statuto, essendo esse al di fuori del tempo, dello spazio e della causalità. L'aggettivo adeguato completa il primo termine della definizione schopenhaueriana, unmittelbare und adäquate Objektität. Adeguato è qui utilizzato alla maniera di Spinoza ${ }^{20}$ : esso sta per "correlato a" la volontà; la volontà in sé si dà una forma, si rende conoscibile attraverso le idee. L'idea, quindi, è posta immediatamente e simultaneamente alla volontà, ovvero si trova di fronte alla volontà.

Stando allora a quanto scrive Schopenhauer, la volontà si rappresenta immediatamente [unmittelbar], ovvero senza mediazione, nelle idee. Si può, allora, sostenere che fra cosa in sé e idee non esiste un medium, un termine medio, l'idea è il "primo grado" di oggettivazione, è la prima maniera possibile di oggettivarsi della volontà: «essa è l'oggettità più adeguata possibile [die möglichst adäquate Objektivität]», tenuto per fermo che essa è toto genere diversa dal Wille an sich.

Qui forse può tornare utile precisare che cosa Schopenhauer intenda con il termine "obbiettivare" e "oggettivare" [objektiviren]: si tratta del passaggio graduale che la Volontà metafisica in sé, entrando nel fenomeno [in Erscheinung eintreten], compie dal generale all'individuale fenomenico per divenire in ultimo grado un oggetto individuale, passaggio che si manifesta chiaramente nell'uomo ${ }^{21}$. Si tratta quindi di un processo graduale e indefinibilmente sfumato. Più la volontà si oggettivizza, più essa diviene individuale. La prima forma di oggettivazione della volontà in sé è la sua manifestazione come fenomeno nella sua forma più generale. E l'unico passaggio immediato possibile

\footnotetext{
${ }^{20}$ SPINOZA, B. Etica dimostrata secondo l'ordine geometrico, Bollati Boringhieri, parte II «Natura e origine della mente», prop. 34: «Ogni idea, che in noi è assoluta, cioè adeguata e perfetta, è vera. / Dimostrazione. Dicendo che in noi si dà un'idea adeguata e perfetta, noi non diciamo altro (per il corollario della prop. 11 di questa parte), se non che Dio, in quanto costituisce l'essenza della nostra mente, si dia un'idea adeguata e perfetta, e di conseguenza (per la prop. 32 di questa parte), non diciamo altro che tale idea sia vera». Cfr. SCHOPENHAUER, A. WWV II/MVR II, capitolo 29, pp. 1682-3: «L'idea è la radice di tutte queste relazioni e quindi il compiuto e perfetto fenomeno, ovvero, come mi sono espresso nel testo, l'adeguata oggettità della volontà in questo grado del suo manifestarsi».

${ }^{21}$ Schopenhauer precisa che, a livello individuale, questo processo di oggettivazione dalla volontà alla coscienza avviene attraverso la percezione [Wahrnehmung]: «Ad ogni modo però la percezione, in cui noi conosciamo i moti e atti della nostra volontà, è di gran lunga più immediata di ogni altra: essa è il punto in cui la cosa in sé entra nel fenomeno [wo das Ding an sich am unmittelbarsten in die Erscheinung tritt] con la massima immediatezza e viene illuminata più da vieino dal soggetto conoscente», SCHOPENHAUER, A. WWV II/MVR II, cap. 18, pp. 1376-7. 
dalla volontà come noumeno al fenomeno sono le idee. Schopenhauer descrive, così, in quale rapporto la volontà si trova di fronte alle idee: le idee sono poste dalla volontà in sé come correlato oggettivo, potremmo dire, con una terminologia hegeliana, per sé. Ma in quale modo la volontà pone le idee? Forse per emanazione, oppure come creatio ex nihilo? Schopenhauer non dà alcuna risposta esplicita al problema. L'unica cosa che ci è dato di sapere è che le idee sono toto genere diverse dalla volontà in sé, sicché possiamo solo pensare, traendone le dovute conseguenze, che le idee, in quanto fenomeni, sono ricavate dalla Volontà in sé ex contrario, come necessario correlato complementare della cosa in sé che, in linea con i principi della Filosofia elementare di Karl L. Reinhold, Schopenhauer intende come toto coelo diverse dalla volontà noumenica.

In ogni caso, per Schopenhauer, la conoscenza umana è incapace di cogliere la Volontà in sé e, quindi, la relazione fra essa e le idee. L'unica maniera in cui le idee sono attingibili dall'uomo è attraverso la loro contemplazione, la quale non è conoscenza razionale ma una "forma altra"22, alternativa, di conoscenza. Le idee si manifestano come correlato oggettivo, adeguato e immediato della volontà in sé. L'unico procedimento per poter spiegare il funzionamento della volontà è quello non discorsivo, per analogia. Essendo esse oggettità adeguata, le idee sono analogon del Wille. Schopenhauer utilizzerà anche in seguito il metodo dell'analogia, sebbene in modo differenziato ${ }^{23}$, per generalizzare a tutti gli enti del mondo i risultati della intuizione della volontà attraverso il corpo, Leib, stabilendo che l'uomo non è solo soggetto logico conoscitivo, ma anche soggetto volitivo.

Ma in che senso l'idea è la forma generale del fenomeno?

Essa ha semplicemente deposto le forme subordinate del fenomeno, le quali tutte noi comprendiamo sotto il principio di ragione, o piuttosto

\footnotetext{
22 «Il riconoscimento di una sfera dell'essere inaccessibile alla conoscenza, costituentesi oltre il mondo, che rimane invece oggetto adeguato della filosofia, è il compromesso a cui Schopenhauer giunge, nello sforzo di conciliare il suo ideale di filosofia con un'esperienza, che in esso non si lascia circoscrivere», in RICONDA, G. Schopenhauer interprete dell'Occidente, pp. 30-31.

${ }^{23}$ In riferimento alle diverse applicazioni del metodo dell'analogia nel sistema filosofico schopenhaueriano, si veda KOßLER, M. Substantielles Wissen und subjektives Handeln, dargestellt in einem Vergleich von Hegel und Schopenhauer, in particolare le pp. 112-119, in cui l'autore distingue diversi livelli di rapporto analogico. Secondo Melandri, del metodo dell'analogia, a partire da Schopenhauer, si sarebbe servito lo stesso Sigmund Freud: «Così Freud vede nel "dolore" il Mittelding che unisce le cose: la forza e lo sforzo, l'oggettivazione prima e l'investimento di energie inibitorie che a tal scopo vien richiesto a nostre spese. [FREUD, Sigmund, Das ich und das Es, in Ges. Werke, vol. XIII, S. 250: «Auch der Schmerz kann unbewusst bleiben, dies Mittelding zwischen äußerer und innerer Wahrnehmung»] A questo riguardo Schopenhauer ha già detto tutto», in MELANDRI, E. La linea e il circolo. Studio logico-filosofico sull'analogia, III, § 118, pp. 811-2. 
non è ancora entrata in esse; ma ha conservato la forma prima e più generale, quella della rappresentazione in genere, dell'essere oggetto per un soggetto 24 .

\section{Idee e specie}

Le idee sono definite da Schopenhauer anche come species rerum, forme archetipe, essenze non commiste a elementi empirici e non derivate da essi. In qualche modo, l'impianto platonico della dottrina delle idee in Schopenhauer sembra risentire della concezione spinoziana della mens aeterna, rispetto alla quale ogni oggetto diviene immagine della divinità. D’altra parte, Schopenhauer sembra ispirarsi anche alla dottrina goethiana delle Grandi Madri, essenze archetipe e matrici del mondo. Dottrina cui il grande poeta di Weimar intese assegnare successivamente una forma scientifica, nella Teoria dei colori, attraverso il concetto dell'Urphaenomen, fenomeno naturale originario e conoscibile, unità simbolica universale, generatrice delle molteplici forme della natura ${ }^{25}$, che sviluppa il tema poetico della forma come entelechia della forza originaria della vita, ovvero con le parole di Goethe, «Geprägte Form, die lebend sich entwickelt» 26 .

Inoltre, Schopenhauer ha affermato che tutti i fenomeni sono determinati sotto il rispetto del tempo, dello spazio e della causalità. Tuttavia, le idee sono, per Schopenhauer, extratemporali ed extraspaziali. Ma allora, in che senso esse appartengono all'ambito del fenomeno? Come può l'idea considerarsi fenomeno generale? In riferimento alla dottrina platonica, nel terzo libro del Mondo Schopenhauer aggiunge che le idee, in quanto gradi oggettivati della volontà, sono

le specie determinate, ovvero le forme e proprietà originarie, non mutevoli, di tutti i corpi naturali, tanto inorganici quanto organici, come pure sono le forze universali che si manifestano secondo leggi

24 SCHOPENHAUER, A. WWV I / MRV I, § 31, p. 357.

25 Cfr. BARBERA, S. Il mondo come volontà e rappresentazione, cit., pp. 125-6: «Non v'è dubbio che per Schopenhauer l'idea, in quanto dichiarato corrispettivo del soggetto inteso come mens aeterna, corrisponde alla spinoziana cosa concepita sub specie aeternitatis; ma occorre inoltre sottolineare che le tre caratteristiche individuali dell'idea - la sintesi di universalità e particolarità, il carattere intuitivo, la natura di archetipo generatore di nuovi fenomeni - corrispondono a quelle che Goethe assegnava nella Teoria dei colori al suo "fenomeno originario". La specificità del fenomeno originario sta nella sua natura di simbolo, ossia di fenomeno particolare che assume un valore universale». Barbera ha sottolineato la valenza simbolica delle idee e la loro derivazione dal gusto estetico neoclassico di Schopenhauer.

${ }^{26}$ Goethes Werk im Kontext (2005). (C) Karsten Worm - InfoSoftWare 2005. Buch: Gedichte. Kapitel: Gott und Welt. Basis-Ausgabe: Berliner Ausgabe, S. BA01:549. E da Goethe, si potrebbe aggiungere, Schopenhauer deriva anche il primato dell'intuizione sull'astrazione.

Le idee: la struttura fenomenologica e astorica del mondo 
universali ${ }^{27}$.

L'idea quindi è anche specie naturale, non logica, come possono esserlo i concetti che infatti è possibile conoscere attraverso la ragione discorsivo-astrattiva. L'idea, diversamente dal concetto, non deriva dall'esperienza, ma la precede. Più precisamente, le idee sono specie in quanto esse

si presentano nella conoscenza dell'individuo legata alla forma del tempo come le specie, ossia come gli individui uniti dal legame della generazione, consecutivi e della stessa natura, e che quindi la specie è l'idea ( $\varepsilon^{\prime \prime} \delta \mathrm{ov}$, species) dispiegata nel tempo ${ }^{28}$.

Idea platonica e specie coincidono de facto, pur non essendo propriamente la medesima cosa, in quanto entrambe sono espressione delle proprietà archetipe ed essenziali degli individui, solo che l'una è l'espressione intuitiva di tali proprietà in quanto forme eterne, l'altra è da intendersi come la manifestazione di tali proprietà in un tempo infinito. L'idea platonica è la matrice extratemporale attraverso la quale la volontà si obiettiva negli individui appartenenti alla stessa classe. In questo senso, Schopenhauer afferma che la specie è

il correlato empirico dell'idea. L'idea è propriamente eterna, la specie invece di durata infinita sebbene il suo manifestarsi su un pianeta si possa estinguere ${ }^{29}$.

Per Schopenhauer, se anche tutti gli individui di una specie si estinguessero (il manifestarsi dell'idea), l'idea della specie continuerebbe ad esistere. Inoltre, l'uomo potrebbe conoscere la specie solamente se potesse fare esperienza di tutti gli individui che la compongono: viventi, passati e futuri.

Tuttavia, sia che si contemplino le idee (intuizione estetica), sia che si proceda dagli individui alle idee (astrazione), le proprietà originarie e comuni degli individui di una stessa specie rimangono le medesime e, quindi, idea e specie corrispondono nella

${ }^{27}$ SCHOPENHAUER, A. WWV I/MVR I, § 30, pp. 346-7.

${ }^{28}$ SCHOPENHAUER, A. WWV II/MVR II, capitolo 42, pp. 1952-3.

${ }^{29}$ SCHOPENHAUER, A. WWV II/MVR II, capitolo 29, pp. 1684-5. Schopenhauer sembra sviluppare un argomento di Sesto Empirico, che Schopenhauer conosceva bene, secondo cui: «[...] se si toglie il genere, si tolgono anche tutte le specie insieme ad esso, mentre, se si toglie la specie, non viene eliminato anche il genere, giacché la specie dipende dal genere e non viceversa», SESTO EMPIRICO, Adversus mathematicos, X 269-275.

Le idee: la struttura fenomenologica e astorica del mondo 
forma, sicché «l'idea è propriamente eterna, la specie di durata infinita» ${ }^{30}$. Non derivando dal mondo empirico, è indifferente che le specie siano "entrate nel fenomeno" come genus, si siano cioè manifestate attraverso la loro obiettivazione, si siano prodotte o riprodotte oppure si siano estinte. Esse preesistono al mondo empirico, la loro essenza archetipa è indifferente alla loro realizzazione nel mondo come fenomeno.

\section{La volontà nella natura: le idee come leggi e forze naturali}

Le idee sono generatrici e legislatrici degli eventi naturali, ma non sono individualizzate o individualizzabili. Come è possibile che si dia un fenomeno che ad un tempo si trova nell'ambito fenomenico ma che non sia individuale? Si pensi alla forza di gravità: essa è la legge dei gravi che sono attratti dalla massa della terra, secondo il principio dell'attrazione universale dei corpi. Si tratta cioè di una legge valida per tutti i fenomeni individuali, valida all'interno del mondo fenomenico, e tuttavia la stessa legge di gravità non è un fenomeno individuale, bensì una sorta di a priori della natura, in base al quale gli oggetti assumono un comportamento unitario e costante. Come scrive Schopenhauer nella Metafisica della natura:

La forza naturale in se stessa è manifestazione [immediata] della volontà, e come tale non è assoggettata alle forme del principio di ragione. Sta fuori da ogni tempo, e sembra quasi attendere costantemente il prodursi delle circostanze che le consentano di entrare in campo e di impadronirsi d'una data materia scacciando le forze che fino a quel movimento la dominavano ${ }^{31}$.

Le idee rappresentano, allora, la struttura fenomenologica e, al contempo, astorica dei singoli fenomeni naturali, in grado di dare ragione di quelle manifestazioni che non cadono sotto il principium individuationis, che non sono causati da una ratio fiendi, e che quindi non possono essere spiegati come cause attraverso la quadruplice radice del principio di ragion sufficiente. Si tratta di una struttura che chiamo fenomenologica perché, obiettivandosi, la volontà metafisica universale si afferma ex gradu in tutta la natura come fenomeno, senza che però nessuno stadio della sua oggettivazione sia pensato come un momento di un processo storico, né come un

30 SCHOPENHAUER, A. WWV II/MVR II, capitolo 29, pp. 1684-5.

${ }^{31}$ SCHOPENHAUER, A. N/N, p. 102.

Le idee: la struttura fenomenologica e astorica del mondo 
elemento interno ad un movimento dialettico che prevede figure, $\grave{a}$ la Hegel. Ogni grado di fenomenizzazione della volontà è a sé stante e si colloca in un ordine gerarchico che va dal generale al particolare, in maniera infinitesimale, sicché con Aristotele Schopenhauer può affermare che natura non facit saltus. Inoltre, le idee rappresentano una struttura astorica, perché le leggi di natura, in quanto idee, sono fuori dal tempo e dallo spazio, sottraendosi così al divenire e funzionando di fatto come $a$ priori della natura, immanenti alla natura. Proprio per questo motivo le leggi di natura sono eterne e valide per tutti i fenomeni, altrimenti muterebbero anche esse con il divenire storico.

In un altro passo della Metafisica della natura, Schopenhauer asserisce che «La legge naturale è [...] la relazione dell'idea con il suo fenomeno», intendendo per fenomeno l'oggetto determinato. Quindi, Schopenhauer identifica la forza naturale con l'idea non individualizzata e definisce la legge naturale ${ }^{32}$ come una relazione costante fra gli oggetti e l'idea stessa. In base a tale relazione, la legge naturale si dimostra infallibile 33 .

Ogni forza naturale generale ed originaria non è dunque altro, nella sua intima essenza, che l'oggettivazione della volontà ad un grado inferiore; noi chiamiamo ciascuno di questi gradi una idea eterna nel senso che Platone ha dato a questa parola. La legge naturale, invece, è la relazione dell'idea con la forma del suo fenomeno ${ }^{34}$.

La forza di gravità, quella di attrazione o d'impenetrabilità dei corpi, così come il magnetismo e l'inerzia, sono tutte idee della volontà, la quale si manifesta nella natura attraverso di esse, nella maniera più generale ovvero non individualizzata. «Queste idee platoniche non sono effetto o causa - come spiega Vecchiotti -, ma condizioni di tutte le cause e di tutti gli effetti. Non c'è, ad esempio, causa dell'elettricità: questa è una forza

\footnotetext{
$32 \mathrm{~N} / \mathrm{N}$, p. 97: «Questa compiuta unità dell'essenza di una forza naturale in tutti i suoi fenomeni, questa invariabile costanza con cui le sue estrinsecazioni si producono, non appena, in base al filo conduttore della causalità, vi siano le condizioni richieste, si definisce, propriamente, legge naturale». Cfr. WWV I, § 26, pp. 284-5: «L'infallibilità delle leggi naturali ha, se si parte dalla conoscenza del particolare e non da quella dell'idea, qualcosa di sorprendente, anzi talvolta quasi di raccapricciante. [...] Invece, se abbiamo penetrato la nozione filosofica per cui una forza naturale è un determinato grado di oggettivazione della volontà, [...], se a noi, dico, è divenuta chiara questa nozione, l'intimo senso della dottrina di Kant, [...] allora comprenderemo che quello stupore per la regolarità e puntualità dell'agire di una forza naturale, per la perfetta uguaglianza di tutti i suoi milioni di fenomeni e per l'infallibilità del loro presentarsi, è in realtà da paragonare allo stupore di un fanciullo o di un selvaggio che, guardando magari per la prima volta un fiore attraverso un vetro dalle molte sfaccettature, si stupisca della perfetta uguaglianza degli innumerevoli fiori che vede e conti uno ad uno i petali di ciascuno di essi».

${ }^{33}$ Cfr. N/N, p. 97. Cfr. VECCHIOTTI, I. Introduzione a Schopenhauer, cit., p. 47: «questo amore [teoretico per Platone] si traduce e si converte quasi nell'idoleggiamento platonico, come l'infallibilità delle leggi naturali quasi si converte nell'immobilità delle idee».

${ }^{34}$ SCHOPENHAUER, A. N/N, p. 99.

Le idee: la struttura fenomenologica e astorica del mondo
} 
originaria intrinseca alle cose e non è effetto di alcuna cosa, né può essere causa, se non nelle manifestazioni» 35 .

Oltre che essere le leggi di natura, Schopenhauer afferma che le idee sono anche forze naturali:

Se dei fenomeni della volontà nei gradi inferiori della sua oggettivazione, dunque la natura inorganica alcuni vengono in conflitto tra loro, ciascuno volendo, sul filo conduttore della causalità, impadronirsi di una data materia: da questa contesa scaturisce la manifestazione di un'idea superiore, che vince tutte quelle più imperfette esistite in precedenza però in modo da lasciar sussistere la loro essenza su un piano subordinato, accogliendone in sé un analogon; un fatto, questo, che è comprensibile appunto solo in base all'identità della volontà che si manifesta in tutte le idee e al suo tendere a un'oggettivazione sempre più alta ${ }^{36}$.

Riprendendo il tema della lotta per la vita e trasferendo il Kampf um die Existenz anche al mondo inorganico, Schopenhauer identifica le idee con tutte le forze della natura, financo le forze atomiche che lottano l'una contro l'altra, ininterrottamente. Da una tale lotta si producono, come risultanti del parallelogramma delle forze, nuove idee superiori. In questo senso le idee superiori sono l'analogon di quelle inferiori, il loro correlato oggettivo: le idee inferiori cioè, come nel parallelogramma delle forze, non cessano di esistere, ma esprimono una forza superiore, la loro risultante, ne sono una sintesi superiore, non già nel divenire storico, ma come elementi dinamici della natura, nella loro simultaneità.

Dalla lotta dei fenomeni della volontà nei gradi più bassi della sua oggettivazione, e cioè all'inorganico [...] scaturisce la manifestazione di una idea più elevata, che sopraffà tutte le precedenti meno perfette, e tuttavia in modo tale da lasciarne sussistere l'essenza in uno stato subordinato, accogliendo in sé un analogon. [...] La volontà unica, che si oggettivizza in tutte le idee, aspira infatti alla più alta oggettivazione possibile e abbandona perciò, dopo un loro conflitto, i gradi inferiori del suo fenomeno, per apparire tanto più potente ad un grado più elevato. Nessuna vittoria senza lotta. L'idea superiore, ossia l'oggettivazione più alta della volontà, dal momento che non può manifestarsi altrimenti che soggiogando le idee inferiori, ne subisce la resistenza ${ }^{37}$.

35 VECCHIOTTI, I. Introduzione a Schopenhauer, cit., p. 46. L'esempio è di Schopenhauer: WWV I/MVR I, § 26, pp. 278-9.

${ }^{36}$ SCHOPENHAUER, A. WWV I/MVR I, § 27, pp. 304-5.

37 SCHOPENHAUER, A. N/N, pp. 114-115.

Le idee: la struttura fenomenologica e astorica del mondo 
E continuando, Schopenhauer sottolinea:

D'altra parte però non bisogna perdere di vista che tutte le idee, ossia in tutte le forze della natura inorganica e in tutte le forme di quella organica, è una sola e medesima volontà che si rivela, cioè che entra nella forma di rappresentazione, nell'oggettità ${ }^{38}$.

Per Schopenhauer, è l'unità del principio metafisico che garantisce l'uniformità dei principi naturali, e assicura che le leggi di natura siano sempre valide e costanti. Inoltre, è sempre l'unità del principio metafisico che rende possibile l'interazione fra le differenti idee, permettendone un rapporto analogico, che altrimenti non sarebbe possibile poiché, come sapevano già gli scolastici, non potest dari relatio sine fundamento, ovvero senza un fondamento non vi è alcuna relazione possibile. In questo senso, con Aristotele, Schopenhauer può affermare non solo che natura non facit saltus,

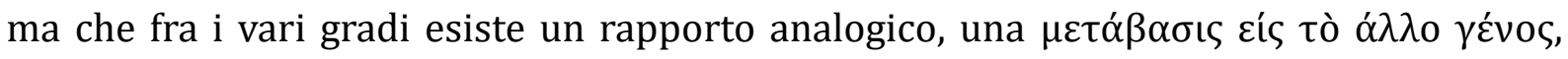
come ripete Schopenhauer con l'Aristotele naturalista del de Coelo, cioè una «traslazione delle forme dell'apparenza nella cosa in sé» ${ }^{39}$. Resta poi che, come ho già avuto modo di scrivere ${ }^{40}$, se da un lato la volontà si manifesta per gradi in senso verticale, sino a raggiungere la sua massima obiettivazione nell'uomo, al punto tale che ogni individuo corrisponde ad una idea specifica ovvero al suo carattere intelligibile, essa si manifesta però anche come criterio orizzontale, ossia unitario dell'intero mondo, poiché principio metafisico di ogni essere vivente.

È vero però che le forze di natura e le leggi non coincidono fra di loro: le prime esprimono la volontà nella sua affermazione generale come fenomeno; le seconde, la relazione fra le idee e i fenomeni. Tuttavia, Schopenhauer non pone una differenza esplicita fra di esse e, quindi, si può dire della prima ciò che si dice della seconda, ovvero che forze e leggi di natura sono idee.

\section{Ideenlehre: Schopenhauer e Natorp}

\footnotetext{
38 SCHOPENHAUER, A. WWV I/, MVR I, § 27, pp. 302-3.

39 SCHOPENHAUER, A. P/P, vol. II, p. 354.

40 «Ogni forza naturale universale e originaria è dunque nella sua essenza intima nient'altro che l'oggettivazione della volontà in grado inferiore. Noi chiamiamo ogni tale grado un'idea eterna, nel senso di Platone. La legge naturale è invece la relazione dell'idea con la forma del suo fenomeno», SCHOPENHAUER, A. WWV I/MVR I, § 26, pp. 286-7.

Le idee: la struttura fenomenologica e astorica del mondo
} 
Se riassumiamo brevemente, per punti, le caratteristiche principali con le quali Schopenhauer definisce l'idea, avremo dunque che

1. l'idea non coincide con la volontà in sé [Wille an sich], vale a dire con la volontà in quanto inattingibile cosa in sé, ancora inespressa ovvero non fenomenizzata, ma le sta di fronte, come correlato oggettivo immediato;

2. l'idea è la forma generale della rappresentazione non ancora individualizzata;

3. l'idea è posta "di fronte alla volontà", ovvero la volontà è di fronte allo specchio delle idee;

4. l'idea è oggettità immediata ed adeguata, «unmittelbare und adäquate Objektität»,

5. il rapporto fra volontà in sé e idee, da un lato, e fra idee e oggetti fenomenici, dall'altro, è di tipo analogico;

6. le idee sono la struttura fenomenologica e astorica del mondo;

7. L'idea è forza e legge di natura.

Una tale caratterizzazione assolve a diverse funzioni del sistema metafisico della volontà universale. Innanzitutto, il mondo delle idee svolge una funzione di mediazione fra volontà noumenica in sé e volontà oggettivata come fenomeno. Senza il mondo delle idee, la volontà infatti si tradurrebbe interamente nel suo processo di oggettivazione, ovvero, essa coinciderebbe con la sua storia, risolvendosi interamente in una sorta di fenomenologia della volontà - à la Hegel. Non di meno, senza il mondo delle idee, vi sarebbero ricadute financo in campo etico. Per esempio, verrebbe meno il principio della responsabilità individuale, poiché è il carattere intelligibile, in qualità di idea individuale dell'individuo, che permette a Schopenhauer - con tutte le contraddizioni del caso che qui non discuto - di affermare che l'individuo risponde delle sue azioni. Inoltre, come «quietivo» della volontà, l'idea consente all'uomo di sottrarsi alla schiavitù della volontà e rende possibile la redenzione per mezzo della contemplazione estetica, rappresentando una sorta di principium indeterminationis, poiché la sua contemplazione scioglie l'individuo dalla catena delle sue determinazioni temporali, spaziali e causali.

Infine, in quanto espressioni e rappresentanti delle forze e delle leggi di natura, le idee sono indeterminate ed oggettive al contempo, esse rappresentano cioè la struttura fenomenologica e astorica dei fenomeni del mondo, senza la quale non vi sarebbero leggi 
valide sempre ed in ogni luogo.

Se considerata nei suoi elementi caratteristici, la Ideenlehre di Schopenhauer si presenta allora come una dottrina originale, che non ha precedenti, perché intende le idee non come enti trascendenti dell'iperuranio, né come espressioni di potenze schellinghiane, incluse come momenti interni alla dialettica della natura e quindi definibili come enti, i quali si esplicano nel loro divenire. Per Schopenhauer, invece, si tratta di modelli eterni immanenti alla natura, sovra- (ante rem) e meta-storici (in re), i quali costituiscono una sorta di intelaiatura a priori metafisica, immanente al mondo, che regge e disciplina le leggi naturali. In questo senso, la dottrina schopenhaueriana delle idee risponde alla ineludibile necessità di dover spiegare, in termini scientifici, l'immutabilità e l'immanenza delle leggi e delle forze naturali. Intendendo presentarsi come una filosofia dell'esperienza ${ }^{41}$, il sistema filosofico schopenhaueriano deve quindi tener conto anche dei fenomeni non individualizzabili, che sfuggono al principio di ragione, e ricomprendere tali leggi e tali forze nella metafisica della volontà, spiegarli cioè alla luce di un principio astorico, immanente e immutabile. Infine, da un punto di vista puramente estetico, se la Volontà è la terribile testa di Medusa che l'uomo non può guardare direttamente in volto, le idee rappresentano allora lo scudo di Minerva, all'interno del quale, come nello specchio di Perseo, il Wille si riflette e permette all'individuo di essere contemplato - solo per riflesso - dalle idee. E come di fronte allo sguardo di Medusa, così la volontà si pietrifica ex gradu, prima nelle forme eterne delle idee e poi via via nelle singole cose, delle quali la volontà rimane l'essenza ultima e metafisica.

Si noti che Schopenhauer si avvale pienamente di tutta la ricchezza semantica del lessico platonico relativo al mondo delle idee ${ }^{42}$, riprendendo l'etimologia dell'žı $\delta o v$, sviluppando il primato della vista sugli altri sensi, talché il genio divine il puro occhio del mondo e il limpido specchio dell'oggetto. E se le idee rappresentano lo specchio della volontà, le cose allora non sono che i pallidi riflessi della volontà attraverso le idee, in

\footnotetext{
41 «Essa [la filosofia di Schopenhauer] non si arroga [...] di spiegare l'esistenza del mondo nelle sue cause ultime; se ne sta, al contrario, ai fatti dell'esperienza esteriore e interiore, quali sono a tutti accessibili, dimostrandone il vero e intimo nesso, senza tuttavia spingersi propriamente al di là, alle cose ultraterrene e ai loro rapporti col mondo. Non fa dunque illazioni su ciò che esiste al di là di ogni esperienza possibile, ma fornisce solo l'interpretazione di quel che è dato nel mondo esterno e nell'autocoscienza, si accontenta dunque di comprendere l'essenza del mondo nella sua interna coerenza con se stessa. Essa è pertanto immanente, nel senso kantiano della parola», SCHOPENHAUER, A. WWV I/MVR I, p. 899.

42 Non a caso, VEGETTI ha parlato di un "dizionario eidetico", Id., Quindici lezioni su Platone, cit., p. 160.

Le idee: la struttura fenomenologica e astorica del mondo
} 
una metafora emanazionistica che ben si concilia anche con la tendenza schopenhaueriana a interpretare l'intuizione estetica come una forma di contemplazione mistica, alla maniera di Plotino.

Sulla base di tutte queste considerazioni, allora, si può forse tentare di stabilire un parallelo, finora intentato ${ }^{43}$, fra la Ideenlehre di Schopenhauer e la lettura delle idee platoniche offerta da Paul Natorp ${ }^{44}$. Ma cosa autorizza un tale passo?

Per quanto i riferimenti espliciti a Schopenhauer da parte di Natorp siano pochi ed egli esprima, fra l'altro, un giudizio sprezzante sulla filosofia schopenhaueriana, definendola una Weltphantasie ${ }^{45}$, Natorp la conobbe certamente molto bene, ed esiste più di un'affinità fra le due interpretazioni della dottrina platonica delle idee, come ci apprestiamo a dimostrare. Ovviamente, prima di stabilire analogie e differenze fra le due teorizzazioni, va posta una fondamentale differenza fra il sistema metafisico schopenhaueriano e quello del filosofo di Marburgo: Natorp, uno dei massimi rappresentanti della scuola neocritica di Marburgo, esclude categoricamente tutta la metafisica della volontà di Schopenhauer, ovvero la metafisica nel senso classico del termine.

Tuttavia, a Schopenhauer Natorp si ricollega, per così dire, anche per via genealogica. L'interpretazione della dottrina platonica delle idee di Natorp si iscrive nel solco di quella tradizione postkantiana, alla quale Schopenhauer appartiene a pieno titolo. Tradizione che, attraverso Friedrich Albert Lange, si è sviluppate nel filone criticista neokantiano della scuola di Marburgo, passando per Hermann Cohen, lo stesso Paul Natorp sino a giungere a Ernst Cassirer.

Qui non si intende discutere se l'interpretazione schopenhaueriana o quella natorpiana delle idee come a priori oggettivi siano fedeli o meno alla lettera e allo spirito di Platone, - la qual cosa sembra discutibile - né liquidare, come fa Gadamer in riferimento a Natorp, una tale interpretazione come «una delle tesi più paradossali che

\footnotetext{
${ }^{43}$ Un vago riferimento è presente in Un percorso da Kant a Natorp, in CHIARADONNA, R. Il platonismo e le scienze, pp. 165-191.

${ }^{44}$ NATORP, P. Platos Ideenlehre. Eine Einführung in den Idealismus, von Paul Natorp, Professor der Philosophie an der Universität Marburg. Zweite, durchgesehene und um einen metakritischen Anhang vermehrte Ausgabe, Verlag von Felix Meiner, Leipzig 1921, S. 572 (la prima edizione, sempre stampata a Leipzig, era stata pubblicata nel 1903). Id., NATORP, P. Logos-Psyche-Eros - Metacritica alla Dottrina delle Idee, Vita e Pensiero, che raccoglie l'Appendice metacritica al Platons Ideenlehre, pp. 457-513, e il Catalogo dei concetti e dei nomi, pp. 535-571.

${ }^{45}$ NATORP, P. Kant oder Herbart, in Sozialpädagogik: Theorie der Willenserziehung auf der Grundlage der Gemeinschaft, p. 54. Una definizione simile era stata data anche dallo schopenhaueriano Paul Rée il quale, congedandosi dalla filosofia di Schopenhauer, aveva definito la filosofia schopenhaueriana una fantasia sul mondo. Cfr. RÉE, P. Philosophie. (Nachhelassenes Werk), pp. 118-119.

Le idee: la struttura fenomenologica e astorica del mondo
} 
siano mai state avanzate nella storiografia filosofica» ${ }^{46}$. Qui interessa sottolineare la novità del paradigma filosofico e la sua capacità di interpretare l'esperienza possibile in una prospettiva neokantiana.

Veniamo allora al rapporto fra le due dottrine delle idee. Natorp dà diverse definizioni di idee, che possono essere raggruppate sotto tre tipi: le idee sono leggi, posizioni di pensiero e metodi. Vediamole ora analiticamente.

Se già per Schopenhauer fra idee e leggi naturali vi è identità, anche per Natorp, «L'idea esprime il termine, il punto infinitamente lontano, al quale indirizzano le vie dell'esperienza; esse sono perciò le leggi del procedimento scientifico» ${ }^{47}$. Già Hermann Cohen, richiamandosi all'idealismo kantiano, aveva ammesso le idee come "subjektiver Akt des Schauens", nella prospettiva di una loro spiegazione psicologica ${ }^{48}$. Natorp si spinge invece sino ad affermare che le idee hanno un significato logico, non psicologico, e sono da considerarsi come funzioni oggettive, non soggettive, dell'essere ${ }^{49}$. In questo modo, Natorp intende collocare fuori dal soggetto kantiano gli a priori, preservandoli dall'accusa di soggettivismo e garantendone così una validità universale che rinvia all'oggetto.

Si è ricordato che per Schopenhauer l'idea (हैı\&ov, species) «è l'unità frammentata nella pluralità in forza della forma temporale-spaziale della nostra apprensione intuitiva; invece il concetto è l'unità ristabilita dalla pluralità per mezzo dell'astrazione operata dalla nostra ragione» ${ }^{50}$. Dal canto suo, richiamandosi anche egli ad una metafora visiva, Natorp sostiene che «l'idea è ciò che guarda e che, nel suo sguardo, configura, senza

\footnotetext{
${ }^{46}$ GADAMER, H.-G. Maestri e compagni nel cammino del pensiero. Uno sguardo retrospettivo, traduzione di G. Moretto, Queriniana, Brescia, p. 53. Cfr. G. Reale, Storia della filosofia antica, volume II, p. 1 e ss.: «Questa espressione, una volta chiarito che non è platonica, può senza dubbio essere usata, a patto che si intenda non l'a-priori in senso kantiano o neokantiano o in generale idealistico, che è un a-priori soggettivo (sia pure in senso trascendentale), bensì un a-priori oggettivo, l'a-priori che il platonico Rosmini ha rivendicato contro Kant. Le Idee sono infatti realtà oggettive assolute, che, mediante l'anamnesi, si impongono come oggetto della mente. E poiché la mente nella reminiscenza coglie e non produce le Idee, e le coglie indipendentemente dall'esperienza, anche se con il concorso dell'esperienza (noi dobbiamo vedere le cose sensibili uguali per "ricordarci" dell'Uguale-in-sé, e così di seguito), possiamo ben parlare della scoperta dell'a-priori, ossia della prima concezione dell'a-priori nella storia della filosofia occidentale». In realtà Reale dimentica proprio Schopenhauer, il quale aveva già formulato un'interpretazione della dottrina delle idee in senso idealistico ma oggettivo.

${ }^{47}$ NATORP, P. Platos Ideenlehre. Guida all'Idealismo [Platos Ideenlehre. Eine Einführung in den Idealismus], cit., pp. 215-216.

${ }^{48}$ Cfr. COHEN, H. Die platonische Ideenlehre psychologisch entwickelt (in «Zeitsch. f. Völkerpsychol. u. Sprachwissensch», 4. Bd. 1866 S. 403-464) u. „Platos Ideenlehre u. die Mathematik" 1879; Id., «Die Idee selbst wird gedacht als Hypothesis», p. 26.

${ }^{49}$ NATORP, P. Logos-Psyche-Eros - Metacritica alla Dottrina delle Idee, cit., p. 90.

${ }^{50}$ SCHOPENHAUER, A. WWV I/MVR I, § 49, pp. 468-9.

Le idee: la struttura fenomenologica e astorica del mondo
} 
essere essa stessa guardata né configurata» 51 . Essa è perciò più della cosiddetta «unità del molteplice», come Aristotele definisce l'idea di Platone (Metafisica, A 9, 990 b 13). L'idea detiene una forma generale e originaria performativa, poiché essa determina la realtà senza esserne però a sua volta determinata. «L'idea non è quindi semplicemente sguardo sulla totalità, sguardo che penetra tutte le cose singole particolari divisibili, ma sguardo intuitivo e unitario [An- und Einschau] a partire dalla totalità» ${ }^{52}$. In questo modo, Natorp afferma l'impossibilità di giungere all'idea per via discorsiva e, come per Platone e poi per Schopenhauer, indica nell'intuizione (in Platone, intuizione intellettuale) l'unica via per cogliere l'idea, sottolineando al contempo la forza unitaria dell'idea e seguendo in questo il Platone del Parmenide [131-A, 157-C]. Se per Schopenhauer le idee si trovano di fronte alla Volontà in sé come di fronte ad uno specchio, per Natorp, esse si trovano invece di fronte alla coscienza, rappresentando, come per Schopenhauer, l'unità del molteplice, ovvero l'unità dalla quale il molteplice deriva. E forse non è un caso che anche in Natorp la coscienza di memoria reinholdiana si affaccia come principio implicito che soggiace alla rappresentazione.

Per Schopenhauer le idee, in quanto modelli archetipi, sono ante rem, perché non dipendono e non derivano dalle cose individuali come i concetti; tuttavia, in quanto rappresentazione, ovvero in quanto species l'idea è in re, sicché egli può affermare che la species è «il correlato empirico dell'idea. L'idea è propriamente eterna, la specie invece di durata infinita. [...] L'idea è species ma non genus: perciò le species sono opere della natura, i genera opera dell'uomo: sono cioè meri concetti» ${ }^{53}$. Per Schopenhauer, quindi, è almeno possibile immaginare che la species si realizzi nel mondo in un tempo infinito, attraverso una finzione. Per Natorp, invece, che immagina le idee come a priori oggettivi kantiani ${ }^{54}$, e quindi come forme pure della natura, tali forme non possono mai reificarsi,

\footnotetext{
51 NATORP, P. Logos-Psyche-Eros - Metacritica alla Dottrina delle Idee, pp. 20-21. NATORP, P. Dottrina platonica delle idee, cap. I, p. 11: «Molto spesso, a differenza di quanto avviene per हैıठov, con la parola idea bisogna pensare non solo passivamente a ciò che viene visto, all'aspetto che la cosa offre, bensì, perlomeno nello stesso tempo, al vedere, alla vista o allo sguardo, alla visione in quanto attività di colui che guarda. Questa parola era perciò come destinata a esprimere e a fissare nella coscienza la scoperta del Logico [Entdeckung des Logischen] in tutta la sua autentica originarietà e vivente forza motrice, ossia la scoperta della legalità [Gesetzlichkeit], delle leggi in virtù di cui il pensiero configura il proprio oggetto quando, per così dire, dirige lo sguardo verso di esso, quando non lo assume semplicemente come oggetto dato».

52 NATORP, P. Logos-Psyche-Eros - Metacritica alla Dottrina delle Idee, cit., pp. 20-21.

53 SCHOPENHAUER, A. WWV II/MVR II, capitolo 29, pp. 1685.

${ }^{54}$ Secondo NATORP, P. Dottrina platonica delle idee, cit., p. 62: «È questo il risvolto notevole della grande conquista del Menone: la scoperta dell'A-priori. La conoscenza in cui consiste la virtù dev'essere necessariamente conoscenza a priori, deve cioè radicarsi nell'autocoscienza».

Le idee: la struttura fenomenologica e astorica del mondo
} 
ma rimangono funzioni trascendentali. In polemica con Aristotele, che secondo Natorp è artefice del fraintendimento in senso trascendente della dottrina delle idee di Platone, Natorp dichiara:

Una reificazione delle Idee, quale viene presupposta da Aristotele, è non solo estranea, ma completamente inconciliabile con tutto ciò: Il senso della Idea come legge non solo non viene eliminato o ristretto, ma viene (intenzionalmente) percorso fino alla fine, e solo allora correttamente confermato e saldamente fondato 55 .

Per Natorp:

La legge come il vero contenuto della scienza, come il fondamento di ogni esattezza e quindi di tutti i beni, come ciò che a ogni cosa, in particolare e in generale, conferisce la sua «figura», il suo eidos: questo e non altro è il centro in cui si raccoglie l'intera trattazione del Gorgia, la quale, pur nei margini esigui dell'allusione, è tanto profonda quanto di ampia prospettiva. Ci troviamo qui già direttamente sulla soglia dell' "Idea". L'Idea significa infatti la legge, nient'altro. E se ora è appunto questo - la legge - Il fondamento del Bene, si comprende allora perché più tardi l'Idea del Bene entri in scena come la suprema delle Idee - non semplicemente secondo il valore, ma anche secondo l'ordine logico-, come l'Idea delle Idee, come il loro ultimo fondamento ontologico e gnoseologico. Il senso ultimo della legge in generale è quello di unità, di conservazione dell'unità in ogni mutamento e divenire. Detto in termini teoretici generali: La legge è la conservazione dell'unità in quanto punto di vista del pensiero per l'interpretazione della molteplicità, della differenza, per il loro recupero nella conoscenza. In termini cosmologici: La legge è la conservazione del terreno fondamentale dell'Essere in ogni mutamento. In termini etici e politici: La legge è la conservazione del senso e della volontà della legalità nell'individuo, e la conservazione della comunanza che viene in tal modo fondata. Ma per Platone il senso puro e semplice della conservazione è quello del Bene ${ }^{56}$.

Al contempo, per Natorp, le idee sono intese come metodo, «metodo della fondazione radicale delle scienze nel fondamento del pensiero stesso in questo senso spirituale [geistlich]: la loro fondazione logica altro non è che l'ultima parola della “Dialettica" platonica»57. Detto in breve, la dottrina delle idee rende possibile la

\footnotetext{
${ }_{55}$ NATORP, P. Logos-Psyche-Eros - Metacritica alla Dottrina delle Idee, cit., p. 60.

${ }^{56}$ NATORP, P. Dottrina platonica delle idee, cit., p. 72.

${ }^{57}$ NATORP, P. Platos Ideenlehre, Kap. VI „Der Staat“, p. 216: «Die Idee als Methode, als die Methode der

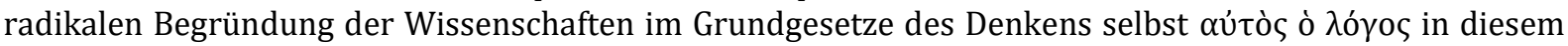
gesteigerten Sinn: ihrer logischen Begründung, das und nichts andres ist das letzte Wort der platonischen „Dialektik"». [tr. It. Dottrina platonica delle idee, cit., p. 267: L'idea come metodo, come il metodo della 
fondazione delle scienze, attraverso la struttura del pensiero, poiché le idee sono «principi logico-gnoseologici» e non già principi soggettivi e antropologici. E anche qui, nemmeno a Schopenhauer era sfuggita l'urgenza di dover trovare nelle idee un principio per fondare le scienze, intese come lo studio delle leggi eterne della natura.

Per Natorp, la sintesi kantiana di materia e forma, il prodotto sintetico delle idee e della natura, è dato invece dal gene (per Schopenhauer, il tipo), dei quali l'idea rappresenta, per dirlo con Natorp, l'unità eidetica - Schopenhauer aveva detto «la specie è l'idea (हैıاov, species)»:

Unità $e$ alterità sono radicati nei geni in quanto tali, dunque in tutti i gene, allora, è con ciò fondata la possibilità - e, nel caso di una attuazione realmente universale, la necessità - dell'individuazione di ogni specie e grado: sicché ogni unità eidetica sarebbe in una molteplicità di individui e sarebbe essa stessa individua, e ogni molteplicità di individui dovrebbe presentarsi in una unità eidetica a sua volta individuale. In tal modo la vita, l'anima, la ragione, la conoscenza avrebbero allora il loro fondamento nell'Idea, così come esige il Sofista ${ }^{58}$.

Infine, per Natorp le idee sono «posizioni di pensiero», poiché «l'essere per Platone è l' "essere del giudizio" ${ }^{59}$, ovvero possiamo parlare di essere solo perché esso è conosciuto, e quindi Natorp, da neokantiano, elimina a monte la dimensione metafisica, che invece per Platone, e poi per Schopenhauer, è fondativa. Ma sebbene Natorp intenda sottrarsi al metodo fondativo della metafisica - e non è detto poi che fino alla fine ci riesca - la tesi che individua nelle idee un che di cognito, anche in questo caso, si dimostra affine a quello schopenhaueriano. Per entrambi, infatti, le idee sono forma generale e quindi condizione postulativa del pensiero nella sua forma non discorsiva, un'altra forma di conoscenza, come aveva sostenuto Platone.

Sin qui, le fondamentali analogie e differenze fra la dottrina delle idee di Schopenhauer e quella di Natorp.

\section{Conclusione}

Si può, infine, sostenere che il parallelo fra la teoria delle idee schopenhaueriana e

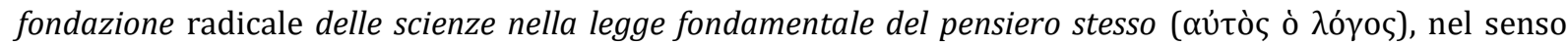
accresciuto di fondazione logica: questa e nient'altro che questa è l'ultima parola della «dialettica» platonica].

58 NATORP, P. Logos-Psyche-Eros - Metacritica alla Dottrina delle Idee, cit., p. 61.

${ }^{59}$ REALE, G. Introduzione. In: NATORP, P. Dottrina platonica delle Idee, pp. XI-XXX: XXI.

Le idee: la struttura fenomenologica e astorica del mondo 
quella natorpiana non solo rende chiara l'originalità della riflessione di Schopenhauer, ma permette anche di segnare una linea di continuità ideale fra Schopenhauer e i neokantiani. Lungi dall'essere licenziata semplicemente come una dottrina estetica romantica, la Ideenlehre schopenhaueriana sembra assolvere invece ad un'esigenza più ampia di scientificità richiesta dalla metafisica dell'esperienza, come essa pretendeva di essere sin da principio. Se è vero, allora, che la Ideenlehre rappresenta uno dei capisaldi della metafisica schopenhaueriana, fondamentale espressione filosofica dell'estetica romantica dell'Ottocento, una tale lettura va però integrata e completata con la considerazione che la dottrina delle idee di Schopenhauer riveste, in senso illuministico, un ruolo fondamentale per la spiegazione dei fenomeni naturali non individualizzabili, e cioè essa si pone come chiave interpretativa epistemologica, in quanto struttura fenomenologica astorica del mondo, in grado di spiegare le leggi e le forze naturali.

\section{Riferimenti bibliografici}

ADOLF, G. Platonic Dialectics and Schleiermacher's Thought: an Essay towards the Reinterpretation of Schleiermacher. Diss. Yale University, 1953.

ARNDT, A. Friedrich Schleiermacher als Philosoph. De Gruyther, 2013.

ASMUTH, C. Interpretation - Transformation, Das Platonbild bei Fichte, Schelling, Hegel, Schleiermacher und Schopenhauer und das Legitimationsproblem der Philosophiegeschichte. Göttingen: Vandenhoeck \& Ruprecht, 2006.

BARBERA, S. Il mondo come volontà e rappresentazione di Schopenhauer: introduzione alla lettura. Roma: Carocci, 1998.

CASSIRER, E. Storia della filosofia moderna, 5. Fichte, Schelling, Hegel, Schopenhauer dai postkantiani alla grande filosofia idealista, Newton Compton Editori, 1978.

CHIARADONNA, R. Il platonismo e le scienze, Università Degli Studi Roma Tre, pp. 165-191.

CIRACÌ, F. Il mondo come volontà, idee e rappresentazione. Per una possibile lettura in senso illuministico della dottrina delle idee. Revista Voluntas: estudos sobre Schopenhauer, Rio de Janeiro, I sem. 2010, vol. 1, n. 1, pp. 71-115.

COHEN, H. Die platonische Ideenlehre psychologisch entwickelt. Zeitsch. f. Völkerpsychol. u. Sprachwissensch, 4. Bd. 1866 S. 403-464.

D'ALFONZO, M. V. (Hrsg.). Schopenhauers Kollegnachschriften der Metaphysik- und Psychologievorlesungen von G. E. Schulze, Göttingen, 1810-11.

GADAMER, H.-G. Maestri e compagni nel cammino del pensiero. Uno sguardo retrospettivo. Traduzione di G. Moretto. Brescia: Queriniana.

GADAMER, H.-G. Schleiermacher als Platoniker. In: Kleine Schriften. Bd. 3. Tübingen, 1972. GIAMETTA, S. Il mondo di Schopenhauer. Verità ed errori. In: SCHOPENHAUER, A. Il mondo come volontà e rappresentazione. Testo tedesco a fronte, a c. di S. Giametta. Milano: Bompiani, 2006, pp. V-LXIII.

GOETHE, J. W. v. Goethes Werk im Kontext. (C) Karsten Worm - InfoSoftWare 2005. Basis-Ausgabe: Berliner Ausgabe, 2005.

HARTMANN, E. Philosophie des Unbewussten. Berlin: Dunker, 1869, 1904XXI. 
HÜBSCHER, A. Arthur Schopenhauer: un filosofo controcorrente. A cura di Giuseppe Invernizzi. Milano: Mursia, 1990.

HÜHN, L. (Hrsg.). Die Ethik Arthur Schopenhauers im Ausgang vom deutschen Idealismus (Fichte/Schelling). Beiträge des Internationalen Kongresses der Schopenhauer-Gesellschaft e. V. (Frankfurt a.M.). Freiburg im Breisgau, 5. bis 8. Mai 2005. Würzburg: Ergon-Verl., 2006. INVERNIZZI, G. Invito al pensiero di Schopenhauer. Milano: Mursia, 1995.

KAMATA, Y. Der junge Schopenhauer. Freiburg-München: Alber, 1988.

. Platonische Idee und die anschauliche Welt bei Schopenhauer. Schopenhauer-Jahrbuch, Bd. 70, 1989, pp. 84-93.

KOßLER, M. Substantielles Wissen und subjektives Handeln, dargestellt in einem Vergleich von Hegel und Schopenhauer. Frankfurt; Bern; New York; Paris: Peter Lang, 1990.

MALTER, R. Der eine Gedanke. Hinführung zur Philosophie Arthur Schopenhauer. Darmstadt: Wissenschaftliche Buchgesellschaft, 1988.

MELANDRI, E. La linea e il circolo. Studio logico-filosofico sull'analogia. Mulino, 1868.

MOLLOWITZ, G. Die Assimilation der platonisch-augustinischen Ideenlehre durch Schopenhauer. Schopenhauer-Jahrbuch, Bd. 66, 1985, pp. 131-151.

MORETTO, G. Platonismo e romanticismo. Platone nei Discorsi sulla religione di Schleiermacher. Archivio di Filosofia. Vol. 52, 1984, pp. 233-269.

NATORP, P. Dottrina platonica delle Idee. Una introduzione all'Idealismo. Intr. di G. Reale; trad. V. Cicero. Vita e pensiero, 1999.

. Kant oder Herbart, in Sozialpädagogik: Theorie der Willenserziehung auf der Grundlage der Gemeinschaft. F. Frommann (E. Hauff), 1899.

Platos Ideenlehre. Guida all'Idealismo [Platos Ideenlehre. Eine Einführung in den Idealismus], 1903/21.

NOVEMBRE, A. Il giovane Schopenhauer e Fichte: la duplicità della coscienza. Dissertatio, LecceMainz, 2011. consultabile all'indirizzo http://ubm.opus.hbznrw.de/volltexte/2014/3685/pdf/doc.pdf.

Platonische Idee und Ding an sich. Der junge Schopenhauer als Leser von Tennemanns Geschichte der Philosophie (in print).

POHL, K. Studien zur Dialektik Friedrich Schleiermachers, Diss. Mainz, 1954.

REALE, G. Introduzione. In: NATORP, P. Dottrina platonica delle Idee. Una introduzione all'Idealismo. Intr. di G. Reale; trad. V. Cicero, Vita e pensiero, 1999, pp. XI-XXX: XXI.

Per una nuova interpretazione di Platone alla luce delle "dottrine non scritte". Milano: Bompiani, 2010XXII.

RÉE, P. Philosophie. (Nachgelassenes Werk), Berlin, 1903.

RICONDA, G. Schopenhauer interprete dell'Occidente. Milano: Mursia, 1986.

SEGALA, M. Schopenhauer, la filosofia, le scienze. Pisa: Scuola Normale Superiore Pisa, 2008.

SCHOLTZ, G. Schleiermacher und die Platonische Ideenlehre, in Internationaler SchleiermacherKongreß 1984. Hrsg. v. K.-V. Selge. Berlin - New York, 1985, pp. 849-871.

SCHOPENHAUER, A. Il mondo come volontà e rappresentazione. A c. di S. Giametta, testo tedesco a fronte. Milano: Bompiani, 2006.

. Parerga e paralipomena. 2 voll. A c. di G. Colli, E. Kühn, M. Carpitella. Milano: Adelphi, 19811983.

. Metafisica della natura. A cura di I. Volpicelli. Bari-Roma: Laterza, 2007.

. Arthur Schopenhauers sämtliche Werke. Bd. IX. Hrsg. von Paul Deussen. München: Piper, 1913.

SCHUBBE, D.; KOßLER, M. (Hrsg.). Schopenhauer-Handbuch. Leben-Werke-Wirkung. Stuttgart: Metzler, 2014.

SPINOZA, B. Etica dimostrata secondo l'ordine geometrico. Tr. it. di S. Giametta. Torino: Bollati Boringhieri, 2002.

VECCHIOTTI, I. Introduzione a Schopenhauer. Roma-Bari: Laterza, 1970, 2003XI.

VEGETTI, M. Quindici lezioni su Platone. Einaudi, 2003.

ZINT, H. Schopenhauer und Platon. Schopenhauer-Jahrbuch, Bd. 14, 1927, 14, pp. 17-41. 
Recebido: $28 / 08 / 16$

Received: 08/28/16

Aprovado: 31/10/16

Approved: 10/31/16 